\title{
Los Programas Universitarios de Personas Mayores y el Envejecimiento Activo
}

\author{
Ana M. Ortiz-Colón \\ Universidad de Jaén, Campus las Lagunillas, s/n. Edificio C5-250. 23071, Jaén-España \\ (e-mail: aortiz@ujaen.es)
}

Recibido Dic. 2, 2014; Aceptado Ene. 13, 2015; Versión final recibida Feb. 10, 2015

\begin{abstract}
Resumen
Los Programas de Personas Mayores se constituyen en el momento actual como ejes estratégicos en materia de responsabilidad social en las Instituciones de Educación Superior. El trabajo que se presenta pretende analizar desde una metodología cualitativa a través de la entrevista, en qué medida el Programa ayuda a prevenir la dependencia de los mayores, y como contribuye al envejecimiento activo, desde la perspectiva del profesorado. Los resultados alcanzados indican que el programa favorece la autoestima y la motivación de las personas mayores, También mejora la comunicación y relaciones interpersonales, junto al desarrollo de capacidades emocionales, físicas y cognitivas.
\end{abstract}

Palabras clave: personas mayores, envejecimiento activo, responsabilidad social, programas universitarios, profesores

\section{University Senior Programs and Active Ageing}

\begin{abstract}
Nowadays Specific Programs for Elderly People function as strategic core ideas in terms of social responsibility at Higher Level Educative Institutions. The present work analyses from a qualitative methodology, through an interview, to what extent the Program contributes to prevent the dependency of elderly people and how it contributes to active ageing from the teacher's perspective. The results show that the program benefits elderly people self-confidence and motivation. Also it improves communication and interpersonal relationships, in addition to the development of emotional, physical and cognitive skills.
\end{abstract}

Keywords: elderly people, active ageing, social responsibility, university programs, teachers 


\section{INTRODUCCIÓN}

Desde un planteamiento de responsabilidad social en la atención a las personas mayores en las Universidades nos planteamos el estudio desde dos ejes, por un lado, el envejecimiento activo y la prevención de la dependencia de los mayores y por otro, los Programas Universitarios de Mayores y la formación permanente como favorecedores del envejecimiento activo, la dependencia y el aumento de la calidad de vida (Rosebruck \& Larkin, 2003). El enfoque de envejecimiento planteado en el estudio, hace referencia a la teoría del envejecimiento activo desde el mantenimiento de una vida activa. Rowe y Khan $(1987,1997)$ abordan el envejecimiento con éxito, mientras que otros autores añaden la satisfacción con la vida como elemento clave de un envejecimiento exitoso (Lher, 1982).

En esta línea destacan los trabajos desarrollados por Fernández-García y Ponce (2013) de gestión de casos, basados en el uso de recursos de programas de aprendizaje a lo largo de la vida, orientando los proyectos vitales de los usuarios hacia un envejecimiento con éxito. Los resultados se orientan a los beneficios que se obtienen con esta combinación como son la motivación, autoestima, estado de ánimo y percepción de la salud, lo que supone una mejora de la calidad de vida, prevención y retraso de la dependencia.

Por otro lado, los estudios desarrollados tanto transversales como longitudinales sobre envejecimiento con éxito, destacan como criterios utilizados, la funcionalidad del individuo y la supervivencia. Depp y Jeste (2006), encontraron 10 aspectos principales en 26 estudios revisados, destacando de mayor a menor aparición: funcionamiento cognitivo, satisfacción con la vida y bienestar, participación social/productividad, presencia/ausencia de enfermedad, longevidad, autovaloración de la salud, personalidad, medio ambiente y renta y autoevaluación de un envejecimiento con éxito. Otros estudios (Fernández Ballesteros y Otros, 2008), consideran el envejecimiento con éxito como multidimensional donde prevalecen tanto criterios simples como combinados.

En el marco de la responsabilidad social, un colectivo que requiere una atención especial, es el de los Mayores, como así lo recoge el plan estratégico de las universidades españolas. Los Programas Universitarios de Mayores en las Universidades suponen una apuesta de las Instituciones de Educación Superior en materia de responsabilidad social, constituyendo un vehículo de desarrollo sociocultural tanto en el contexto de la ciudad como de la provincia, o del país. Otros referentes se pueden encontrar en diferentes legislaciones como la Declaración Universal de Derechos Humanos (1948), la Carta Europea de los Mayores (1992), ley de Atención y Protección a las Personas Mayores (1999), la II Asamblea Mundial sobre el envejecimiento (2002), la Carta de Derechos Fundamentales de la Unión Europea (2000), la iniciativa de la Unión Europea, del Memorandum sobre el aprendizaje permanente (2000), la VI Conferencia sobre Educación de Adultos (2009), los Proyectos de Naciones Unidas para 2050 y las distintas cumbres sobre los objetivos de desarrollo del Milenio de la ONU (2010), entre otras.

En este sentido, los Programas Universitarios de Mayores se plantean como una apuesta decidida por la democratización del conocimiento, entendiendo la formación permanente como aprender para saber vivir mejor una vida más larga, en la que la educación es una herramienta más para lograr el estado del bienestar. De este modo, los Programas Universitarios de Mayores se proyectan como favorecedores del envejecimiento activo, la prevención de la dependencia y el aumento de la calidad de vida. Las inquietudes e intereses de los mayores van más allá de la edad, no encontrando limitaciones por aprender ni que condicionen la participación en temas de interés, siendo fundamental para ellos su presencia en la Universidad (Sáez, 2003).

El modelo en el que se inspira el Programa Universitario de Mayores de la Universidad de Jaén, es el modelo francés, basado en el contexto universitario y estructurado en ciclos, cursos y créditos. La formación se basa en la docencia y las líneas de investigación más destacadas de la Universidad. La metodología que fundamenta los programas universitarios de mayores, responde a las teorías del aprendizaje con mayores y al desarrollo cognitivo. Este modelo es el más desarrollado en Europa en países como Francia, Alemania, Italia y España.

Otra dimensión del Programa es el desarrollo intergeneracional de los mayores con los jóvenes, compartiendo materias en las aulas ordinarias. La evolución del Programa en la Universidad de Jaén, nos ha llevado a plantearnos el propósito de conocer los efectos que el Programa Universitario de Mayores tiene en la prevención de la dependencia en las personas mayores, descubriendo qué dimensiones del programa ayudan a favorecer el envejecimiento activo. El objetivo que nos proponemos, partiendo de los interrogantes anteriores es Analizar en qué medida el Programa Universitario de Mayores ayuda a prevenir la dependencia de las personas mayores desde la perspectiva del profesorado, partiendo de los enfoques planteados anteriormente. 


\section{MÉTODOLOGÍA}

En la investigación hemos seguido una metodología cualitativa a través del análisis de contenido de la entrevista semiestructurada (Colás, 2005). El estudio tiene en consideración los presupuestos de la teoría fundamentada, desarrollada originariamente por Glaser y Strauss (1967). Este enfoque resulta útil y nos permite profundizar en las consideraciones del profesorado sobre la formación de los mayores en el marco de los Programas Universitarios en las Universidades, en referencia al envejecimiento activo y prevención de la dependencia, partiendo directamente de los datos. Pretendemos darle autenticidad y veracidad a la información recogida a partir de las reflexiones y respuestas halladas en el estudio (Denzin, 1989). La entrevista semiestructurada, según afirma Buendía (2005), es el tipo más flexible y abierto. El entrevistador posee la libertad de alterar el orden y la forma de preguntar, así como el número de preguntas. Se dispone de un guión base que se puede ir modificando en el transcurso de la entrevista, aunque siempre manteniendo el objetivo para el cual ha sido preparada y los diferentes puntos sobre los que debe obtenerse la información.

Una vez diseñado y revisado el protocolo de entrevista por 10 expertos universitarios en los referidos programas, se procedió a enviarlo a través de correo electrónico a la muestra seleccionada, siendo representativa de la población objeto de estudio (Buendía, 2005). Una vez conocido el protocolo de entrevista por los entrevistados, así como el calendario y el lugar de realización de las entrevistas, se volvieron a enviar éstas a los entrevistados para su negociación final. Las entrevistas en profundidad fueron grabadas en audio y transcritas para su posterior análisis. El análisis de contenido de las entrevistas se realizó con el programa informático Atlas. ti 6.0, para darle un mayor rigor científico a las conclusiones obtenidas, con el fin de clasificar la información obtenida en relación a conceptos abstractos de categorización de categorías (unidades de registro clasificatorias) y la codificación (unidades de numeración), lo que permitió la presentación de datos a partir de resúmenes, esquemas y diagramas. Posteriormente se establecieron relaciones complejas entre códigos y categorías.

La población del estudio está constituida por 317 profesores/as que imparten su docencia en el Programa Universitario de Mayores y la muestra está formada por 32 profesores/as de la Universidad de Jaén, que ha sido seleccionada utilizando el criterio de heterogeneidad, procurando que al menos haya un profesor/a de cada uno de los módulos que componen el programa (Raposo \& Martínez, 2011). El análisis de las entrevistas se trianguló con un Grupo de discusión creado al efecto en el marco de un trabajo más amplio en el que participó tanto profesorado como alumnado del Programa Universitario de Mayores, con la finalidad de conocer la consistencia de los datos, siendo dicho estudio objeto de otro trabajo. En el estudio no se pretende la generalización, sino formular hipótesis de trabajo que guíen el hacer, con el fin de comprender la realidad como un todo integrado y unificado (García Llamas, 2001)

\section{RESULTADOS}

La presentación de los resultados sigue el protocolo que realiza el programa informático Atlas. ti 6.0, presentándose en primer lugar una referencia a la entrevista realizada, seguida del número de la cita, la categoría a la que pertenece, el número de línea en la que aparece la cita, nombre del entrevistado (se obvia), los códigos asignados a la cita (codes: [Cont cotidiano] [Contenidos] [Relevantes] y la cita en concreto.

En relación a lo anterior, la fase de reducción de los datos, consistió en la categorización temática de cada una de las cuestiones planteadas y sus respuestas, para lo cual se definieron 9 categorías en base a las frecuencias obtenidas en las entrevistas de mayor a menor presencia: Contenidos (19), Capacidades (14), Desarrollo personal y autonomía (14), Actitudes (12), Percepción de sí mismas (12), Sociabilidad (12), Cambio de estereotipos y de actitudes (8), Motivación (8) y Desarrollo cognitivo (8), de acuerdo al protocolo seguido y a las respuestas del profesorado entrevistado (ver figura 1). Las categorías a su vez se engloban dentro de tres grandes familias/ dimensiones relacionadas en todo momento unas con otras, interrelacionando de este modo tanto sus códigos como sus categorías. Las familias/dimensiones quedaron establecidas en: Programa Universitario de Mayores, Prevención de la dependencia, Envejecimiento activo (Ortiz, 2013). En total el Programa ha diferenciado 107 citas en el total de las entrevistas (ver figura 2).

El análisis de las entrevistas ha permitido conocer las opiniones del profesorado, destacando los fragmentos que hemos considerado ilustran sus percepciones y que servirán para obtener las conclusiones de la investigación y el grado de importancia que otorgan a las preguntas de la investigación en cuestión. Ante la pregunta formulada sobre los contenidos más importantes a trabajar dentro del Programa Universitario de Mayores, respondían: (...) los relacionados directamente con la vida cotidiana de las personas mayores 0 que les puedan resultar prácticos (P. 3: Entrevista 03.rtf3.1[CONTENIDOS](4:4) Codes [Cont cotidianos] [Contenidos]. 


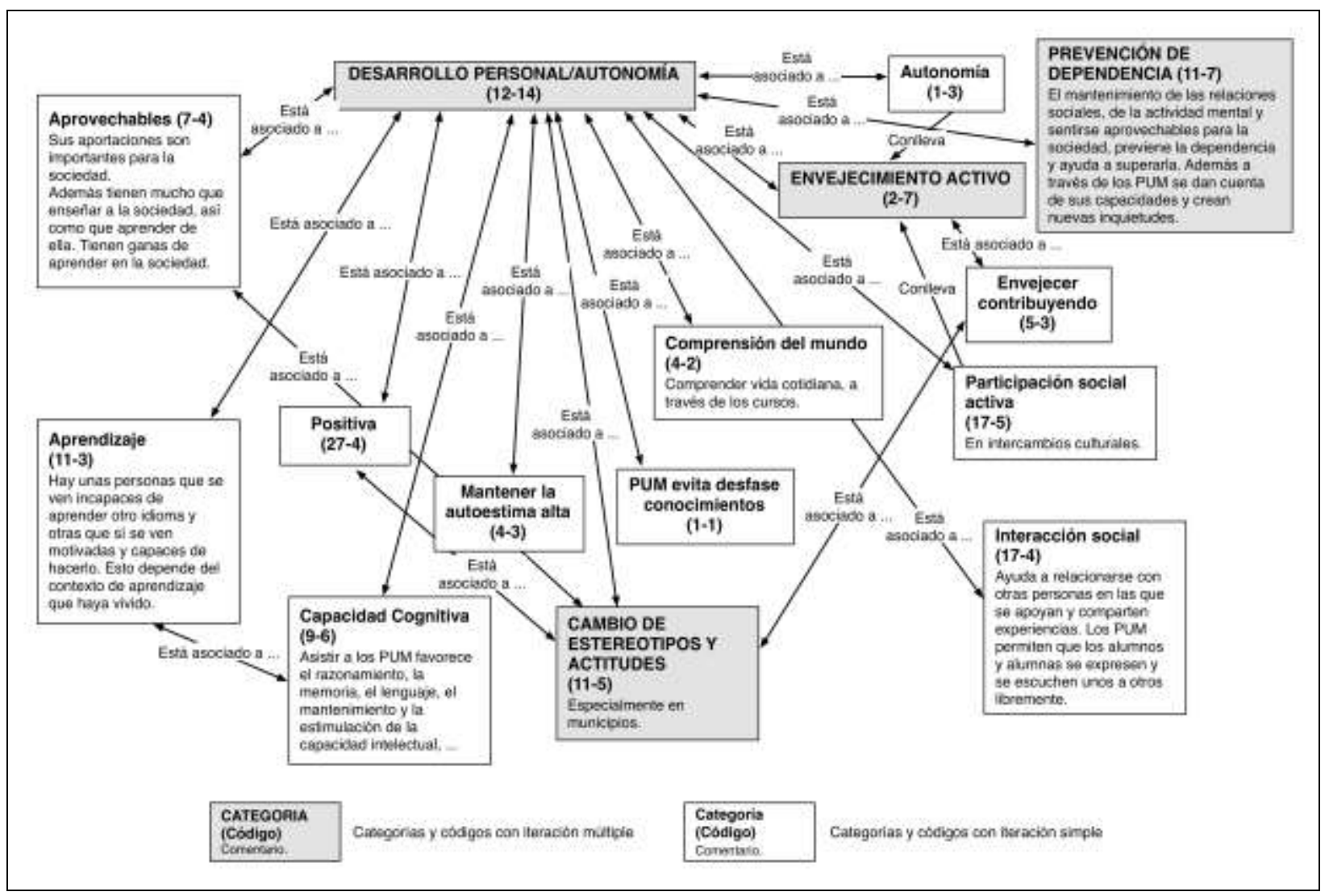

Fig. 1. Mapa de relaciones entre categorías

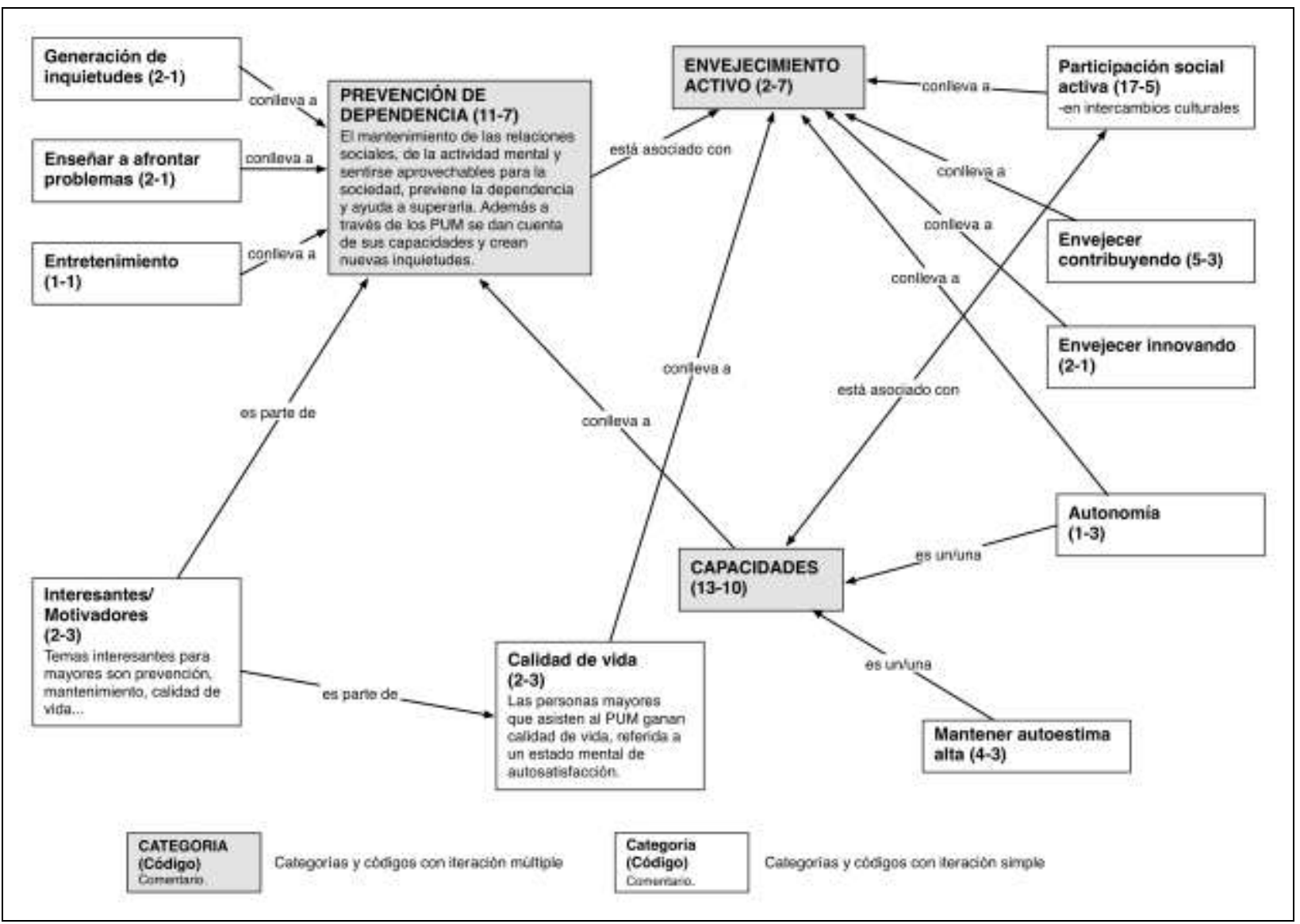

Fig. 2. Mapa de relaciones entre dimensiones 
Ante la pregunta sobre las capacidades que deben mantenerse activas en las personas mayores: Capacidades físicas, cognitivas y emocionales. Físicas, porque favorecen el movimiento y reducen el sedentarismo propiciando un estilo más activo. Cognitivas, porque favorecen el razonamiento, la memoria, el lenguaje, etc. Emocionales, porque proporcionan apoyo social y emocional, favorecen las relaciones con otros y reducen el aislamiento y la soledad P.3. Entrevista 03.rtf- 3.2. [Capacidades] (5:5) Codes: [Capac. Cognitiva] [Capc. Emocional] [Capac. Física] [Capacidades] (ver figura 3).

Referido a cómo se ven los alumnos y alumnas matriculadas en el Programa Universitario para Mayores: Activas P. 3: Entrevista 03. Rtf- 3:3 [Percepción De Sí Mismas] (7:7) Codes: [Activas] [Participación Social Activa] [Percepción De Sí Mismas]. La respuesta planteada a la pregunta sobre si el Programa Universitario para Mayores previene la dependencia y de qué modo, responde: Sí, un estilo de vida más activo y estrategias de afrontamiento diversas ante las contrariedades. P.3: Entrevista 03.rtf- 3:7 [Prevención De Dependencia] (9:9) Codes: [Enseñar a afrontar problemas] (Optimistas) [Participación social activa] [Prevención De Dependencia].

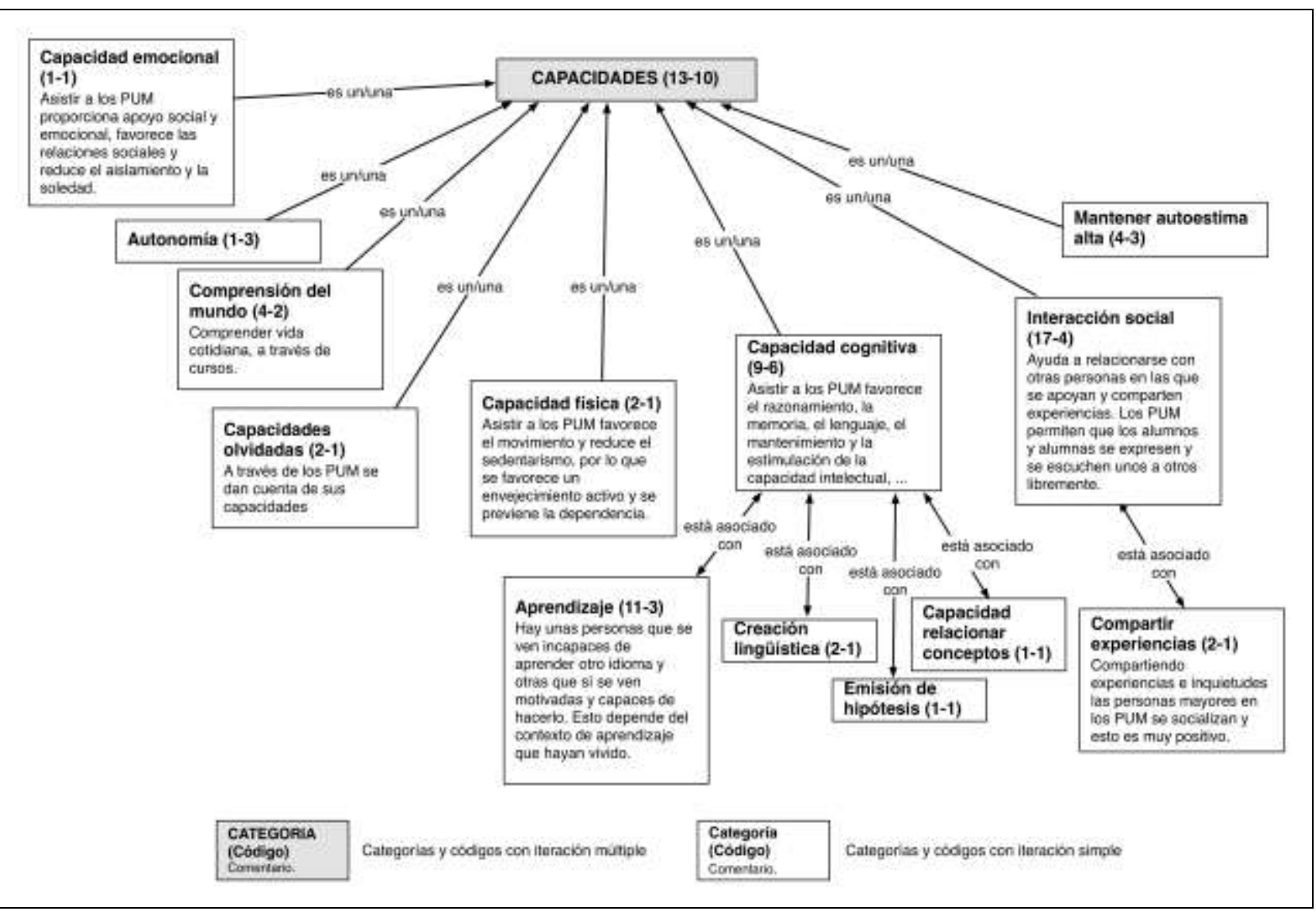

Fig. 3. Mapa de relaciones entre capacidades

En referencia a las capacidades que observan en los mayores, la respuesta expresada en la entrevista P1: Entrevista 01.rtf - 1:7 [Prevención De Dependencia] (9:9) Codes: [Cap Olvidadas] [prevención de dependencia], hace referencia a que no se dan cuenta de las capacidades que tienen y les descubre nuevos ámbitos de interés hacia donde orientar sus inquietudes: capacidades olvidadas, comprensión del mundo, compartir experiencias. En relación a la oportunidad de compartir experiencias podemos observar lo expresado en la entrevista P 3: Entrevista 03.rtf - 3:8 [Sociabilidad] (10:10) Codes: [Compartir experiencias] [Interacción social] [Sociabilidad] Compartir con otros distintas experiencias, con una meta común es una forma de arraigo social muy positiva.

Otra de las respuestas destacadas del profesorado entrevistado, hace referencia a que los alumnos en estas clases son capaces de expresarse libremente, se sienten escuchados por sus compañeros y por el profesorado y de esta forma se potencia su desarrollo personal y su autonomía P10: Entrevista 10.rtf 10:5 [Desarrollo Personal Y Autonomí... (8:8) Codes: [Aprovechables] [Cap Cognitiva] [Desarrollo Personal/Autonomía] [Envejecer contribuyendo] [Interacción social] [Positiva] mantenimiento de una capacidad intelectual importante. 
En relación a la comunicación y relaciones interpersonales que se establecen en los programas universitarios de mayores, destacan las respuestas del profesorado en relación a que los alumnos en estas clases son capaces de expresarse libremente, se sienten escuchados por sus compañeros y por el profesorado P10: Entrevista 10.rtf - 10:11 [Capacidades] (8:8) Codes: [Capacidades] [Comunicación]. Asimismo otro profesor indica las posibilidades de los mayores a la hora de hacer grupos de personas con unas mismas inquietudes, problemáticas y pensamientos. Les ayuda a tener una red social más amplia y en definitiva los hace más felices P 7: Entrevista 07.rtf - 7:7 [Sociabilidad] (10:10) Codes: [Compartir experiencias] [Comunicación] [Enseñar a afrontar problemas] [Generación de inquietudes] [Interacción social] [Sociabilidad].

Las relaciones interpersonales que se establecen con los Programas universitarios de mayores, vienen a cubrir la tendencia al aislamiento en las personas mayores relacionado con los cambios en la dinámica familiar y en la pérdida de las relaciones sociales vinculadas al trabajo. Esta tendencia al aislamiento influye en la pérdida de autoestima, la pérdida del sentido de independencia, etc. P 3: Entrevista 03.rtf 3:9 [Sociabilidad] (10:10) Codes: [Prevención De Dependencia] [Sociabilidad]. Otro entrevistado expresa que los programas, favorecen la relación entre los miembros de una comunidad en un momento clave de sus vidas. P 4: Entrevista 04.rtf - 4:3 [Capacidades] (5:5) Codes: [Capacidades] [Interacción social]

\section{DISCUSIÓN}

De los análisis realizados, se deduce que los entrevistados encuentran esencial el Programa Universitario de Mayores en cuanto al desarrollo de competencias básicas, la prevención del envejecimiento, la resolución de problemas y la calidad de vida entre otros (Villar, 2010).

Haciendo una síntesis de las opiniones más relevantes de cada profesor en referencia a las respuestas dadas, nos indican que los programas de mayores favorecen la autoestima y la motivación de las personas mayores al sentirse útiles, válidas y capaces de aprender y participar de forma activa en la sociedad. Por otro lado destaca la comunicación entre todos los agentes que participan en el Programa de Mayores, permitiendo que se expresen libremente y se escuchen unos a otros, compartiendo sus experiencias, inquietudes, problemáticas, pensamientos; discutiendo, etc. Destaca la importancia del desarrollo de capacidades tanto emocionales como físicas y cognitivas.

Si hacemos un análisis comparativo de las percepciones del profesorado halladas en el estudio, se pueden constatar los planteamientos de Rowe y Khan $(1987,1997)$ y Depp y Jeste (2006) de funcionalidad cognitiva, satisfacción con la vida y bienestar y participación social como los de mayor aparición, obteniendo las máximas frecuencias. Los entrevistados expresan que desde los Programas Universitarios para Mayores se previene la dependencia al tratar de sensibilizar a los mayores hacia un estilo de vida activo, aprendiendo a afrontar problemas, favoreciendo la movilidad y la comunicación con otras personas, estimulando el cerebro, entreteniéndolos, generando inquietudes, enseñando a envejecer activamente.

A partir de las opiniones de todo el profesorado, se confirma lo que Walker (2009) afirma cuando dice que todas las personas independientemente del grado de dependencia que tengan, pueden llegar a conseguir envejecer activamente, siempre y cuando se potencien al máximo sus capacidades y autonomía. El profesorado entrevistado está de acuerdo en afirmar que este programa, potencia y mantiene en los mayores distintas capacidades, entre las que destacan la de aprendizaje, la cognitiva y la de interacción social, muy importantes las tres porque las personas mayores con estas tres capacidades pueden ser perfectamente autónomos y sentirse útiles, para proponerse nuevas metas y envejecer activamente (Pérez Serrano y otros, 2009). El profesorado coincide en expresar la importancia del modelo comunicativo formativo que desarrollan con los mayores, favoreciendo la participación y la interacción en el proceso de aprendizaje desarrollado, en línea con los planteamientos expresados por Lirio y Morales (2011) en referencia a que los mayores aprenden participando y construyendo conocimiento de una forma activa.

Por todo ello, se puede considerar que el Programa Universitario de Mayores, está cambiando las concepciones que la sociedad tiene adquiridas sobre las personas mayores, tanto desde las percepciones del profesorado como desde los resultados alcanzados en un trabajo más amplio con alumnado del Programa Universitario de Mayores de la Universidad de Jaén (Ortiz, 2013). Envejecer de forma activa implica la participación y contribución social activa de la persona mayor en todos sus ámbitos, previniendo de este modo diferentes situaciones de dependencia (Martín y Requejo, 2005). Los Programas Universitarios de Mayores favorecen, por tanto, a los mayores a contribuir en la sociedad, aportando ideas, debatiendo diferentes temas, opinando, innovando, rompiendo con la visión general que la sociedad tiene sobre la vejez; contribuyendo de este modo desde la Universidad a los tres pilares fundamentales sobre los que se levanta el envejecimiento activo: participar, contribuir e innovar (Castaño \& Garín, 2012). 


\section{CONCLUSIONES}

Hemos presentado las actuaciones que en materia de responsabilidad social se desarrollan en las Universidades con las personas mayores analizando en qué medida el Programa Universitario de Mayores de la Universidad de Jaén, ayuda a prevenir la dependencia de las personas mayores, desde la perspectiva del profesorado, según las tesis planteadas en el estudio. Como primera conclusión, el profesorado señala como ventajas, la funcionalidad cognitiva, la satisfacción con la vida y bienestar y la participación social.

Otra conclusión del trabajo desde la perspectiva de profesorado, confirma que los Programas Universitarios de Mayores favorecen la autoestima y la motivación de las personas mayores, junto al desarrollo de capacidades emocionales, físicas y cognitivas, en línea con los trabajos desarrollados por Fernández-García y Ponce (2013) y Pérez Serrano y otros (2009).

Finalmente, otra conclusión del estudio es el significado que los Programas Universitarios de Mayores adquieren para aquellas personas que no han tenido experiencia universitaria a lo largo de su vida, al ser diseñados específicamente para Mayores, confirmándose los resultados obtenidos por Montoro-Rodríguez y Pinazo, 2005) respecto a la participación para la mejora de la vida social y el desarrollo cognitivo, como bases del envejecimiento activo y la prevención de la dependencia.

\section{REFERENCIAS}

Buendía, L., La investigación por encuesta. En Buendía Eisman, L. Colás Bravo, P. y Hernández Pina, F. Métodos de investigación en psicopedagogía. McGraw-Hill, Madrid (España) (2005).

Castaño, M, y Garín, A. Incorporación de Personas Adultas a los Estudios Universitarios: Aprendizaje Permanente para Arquitectos Técnicos. doi.org/10.4067/S0718-50062012000300003. Form. Univ., 5(3), 17-26 (2012).

Colás P., Métodos y técnicas cualitativas de la investigación en psicopedagogía. En Buendía Eisman, L. Colás Bravo, P. y Hernández Pina, F. Métodos de investigación en psicopedagogía. McGraw-Hill, Madrid (España) (2005).

Comisión de las Comunidades Europeas, Memorándum sobre el aprendizaje permanente. COM (2000)1832. Bruselas, (2001).

Depp, C. A. y D. V. Jeste, Definitions and predictors of successful ageing: A comprehensive review of larger quantitative studies. American Journal os geriatric Psychiatry, 14, 6-20 (2006).

Denzin, N. K., Interpretative biography. Sage, Newbury Park (1989).

Fernández-García, T. y L. Ponce, Envejecimiento activo: recomendaciones para la intervención social desde el modelo de gestión de casos. Portularia 13, 1, 87-97 (2013).

Fernández Ballesteros, R., L. García, D. Blanc, A. Efklides, R. Kornfeld, A. J. Lerma, Mendoza, V., N. Mendoza, T. Orosa, C. Paul, y S. Patricia, Lay concept of aging well: Cross-cultural comparisons. J. American Geriatric Society, 56, 950-952 (2008).

García Llamas, J. L. y otros. Introducción a la investigación en educación. Madrid, UNED (2001).

Glaser, B. G. \& Strauss, A.L. The discovery of grounded theory: strategies of qualitative research. Chicago: Aldine, (1967).

IMSERSO, Las personas mayores en España, informe 2006. Instituto de servicios sociales, Madrid (2007).

Lher, U., Socio-Psichologycal correlates of longevit. ISSN: 01988794, Annual Review of gerontoloy and Geriatrics, 3, 102-147 (1982).

Lirio, J. y S. Morales, El reto de la formación el profesorado para una enseñanza de calidad en las universidades de mayores. Pedagogía Social. Revista Interuniversitaria, 19, 155-166 (2012).

Martín, A. V. y Requejo, A., Fundamentos y propuestas de la educación no formal con personas mayores. Revista de Educación, 338, pp. 45-66 (2005). 
Montoro-Rodríguez J y S. Pinazo, Evaluating social integration and psychological outcomes for older adults enrolled at a university intergenerational program. doi/abs/10.1300/J194v03n03_05\#.VMTJrGSG-SU. J. of Intergenerational Relationships, 3, 65-83 (2005).

ONU, Informe de la Il Asamblea Mundial sobre el envejecimiento, Naciones Unidas. Nueva York (2002).

Ortiz, A. M., La formación de personas mayores como favorecedora del envejecimiento y calidad de vida desde las percepciones del profesorado de la Universidad de Jaén. Revista de Ciencias de la educación: Órgano del Instituto Calasanz de Ciencias de la Educación, 234, 239-252 (2013).

Pérez Serrano, G., García, J.L. Sarrate, M.L.,Pérez Serrano, M.,De Miguel, S.,Cuenca, M.E., Evaluación de la calidad de los programas universitarios para mayores: Propuestas de mejora. Programa de Estudios y Análisis 2009. (MICINN) (2009).

Raposo M., y Martínez, E., La Rúbrica en la Enseñanza Universitaria: Un Recurso Para la Tutoría de Grupos de estudiantes. doi: 10.4067/S0718-50062011000400004, Formación Universitaria, 4(4), 19-28 (2011).

Rosebruck, V., y L. Larkin, Introducing Standards and Guidelines. A Rationale for Defining the Knowledge, SKills and Dispositions of Intergenerational Practice. doi/abs/10.1300/J194v01n01_11\#.VMTLE2SG-SU. J.f intergenerational Relationships, 1 (1), 133-144 (2003).

Rowe, J. W. y R. L. Khan, Human aging: Usual and successfull. DOI: 10.1126 / science.3299702. Science, 237, 143-149 (1987).

Rowe, J.W. y R. L. Khan, Sucessfull aging. doi: 10.1093/geront/37.4.433. The Gerontologist, 37, 433-440 (1997).

Sáez, J., Educación y aprendizaje en las personas mayores. Dykinson, Madrid (España) (2003).

UNESCO, La educación encierra un tesoro. Informe de la Comisión Internacional sobre la educación para el Siglo XXI. Santillana, Madrid (España) (1996).

Villar, F., Evaluación de programas universitarios para mayores: Motivaciones, dificultades y contribuciones a la calidad de vida demanda y prestación de cuidados en el seno familiar. http://www.imsersomayores.csic.es/documentos/documentos/imserso-estudiosidi-50.pdf Madrid. Estudios de I+D+I, 50, (2010).

Walker, A., The emergence and application of active aging in Europe.

http://dx.doi.org/10.1080/08959420802529986. Journal of Aging \& Social Policy, 21(1), 75-93 (2008). 